\title{
Transmission-Line Modeling and Sensitivity Evaluation for Lumped Network Simulation and Design in the Time Domain*
}

by J. W. BANDLER

Group on Simulation, Optimization and Control, Department of Electrical Engineering, McMaster University, Hamilton, Canada L8S $4 L 7$

P. B. JOHNS

Department of Electrical and Electronic Engineering, University of Nottingham, Nottingham, England NG7 2RD

and M. R. M. RIZK

Group on Simulation. Optimization and Control, Department of Electrical Engineering, McMaster University, Hamilton, Canada L8S $4 L 7$

ABSTRACT: A new approach for time-domain analysis and design of lumped networks is considered. The lumped elements are modeled by transmission-line sections or stubs and the modeled network is analysed by the transmission-line matrix (TLM) method, which provides an exact solution to the model. Compensation of errors arising in modeling the network elements is discussed. Sensitivities w.r.t. design variables can easily be obtained and thus used in optimization. Sensitivities w.r.t. time and the time step are also obtained and used to improve the model's response.

\section{Introduction}

The transmission-line matrix (TLM) method of numerical analysis provides a new approach to the time domain analysis of lumped networks. The method has previously been extensively used for solving electromagnetic vector field problems in two and three dimensions (1). The technique has also been used for solving the diffusion equation (2).

In its application to lumped networks (3), the TLM method has some advantages because it provides an exact solution to the transmission-line networks used to model the actual networks. The paper demonstrates how the transmission-line models for lumped networks can be derived, and how to compensate for modeling errors in terms of additional network elements.

* This work was supported by the National Research Council of Canada under Grant A7239. This paper was presented at the Conference on Computer-aided Design of Electronic and Microwave Circuits and Systems, Hull, England, July 1977. 


\section{J. W. Bandler, P. B. Johns and M. R. M. Rizk}

The TLM method is easily programmed. Calculation of exact sensitivities for the model w.r.t. design variables is possible. A symmetrical LC lowpass filter has been optimized in the time domain using TLM analysis, the required gradients being obtained from the sensitivities derived in the paper.

Sensitivities with respect to the time step are also derived, from which an approximation to the time sensitivities is obtained. Using these formulas and the TLM results, we can extrapolate to the near exact impulse response.

\section{Transmission-Line Modeling}

The time domain response of a lumped network can be found using the TLM method, after choosing an appropriate transmission-line model for the network. Inductors and capacitors are represented either by transmission lines or by stubs.

\section{Link Modeling}

First consider the modeling of a series inductor and a shunt capacitor, each by a transmission line. To simplify the analysis, certain assumptions must be made. We will let all the transmission line models have the same length, and let the time taken by a pulse to travel along each transmission line be the same, namely, T. The lumped inductor $L$ shown in Fig. 1(a) can have the transmission-line model shown in Fig. 1(b) with an inductance per unit length $L_{d}$, where

$$
L_{d} l=L
$$

The velocity of propagation on the transmission-line may be expressed as

$$
\frac{1}{\sqrt{L_{d} C_{d}}}=\frac{l}{T}
$$

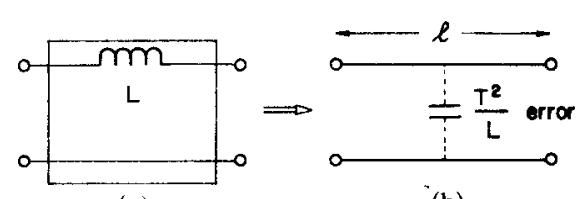

(a)

(b)

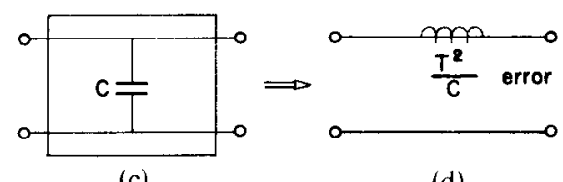

(c)

(d)

FIG. 1. Lossless transmission-line models of a series inductor and a shunt capacitor. 
and hence the distributed capacitance $C_{d}$ is given by

$$
C_{d}=\left(\frac{T}{l}\right)^{2} \frac{1}{L_{d}} .
$$

The basic parameter which determines how pulses are scattered throughout a transmission-line network is the characteristic impedance $Z_{0}$, which for the model of inductor, is obtained from (1) and (3). Thus,

$$
Z_{0}=\sqrt{\frac{L_{d}}{C_{d}}}=\frac{L}{T} .
$$

The error associated with the model of the inductor is due to the distributed capacitance given in (3). This may be approximated in the lumped circuit by a lumped shunt capacitor $C_{c}$ representing the error, which is given by

$$
C_{e}=C_{d} l=\left(\frac{T}{l}\right)^{2} \frac{l}{L_{d}}=\frac{T^{2}}{L} .
$$

This lumped capacitor is shown dotted in Fig. 1(b).

The characteristic impedance for a transmission line modeling a lumped capacitor (Fig. 1(c)) may be derived in the same way, the result being

$$
Z_{0}=\frac{T}{C}
$$

and the error this time will be represented by a series lumped inductor $L_{e}$ (Fig. 1(d)) of value

$$
L_{e}=\frac{T^{2}}{C}
$$

It is clear that if $T$ is small then for the model of the inductor $Z_{0}$ and $L_{d}$ are large while the unwanted shunt distributed capacitance $C_{d}$ is small. On the other hand, for the model of the capacitor $Z_{0}$ and the unwanted $L_{d}$ will be small if $T$ is small. So, as $T$ becomes smaller, the transmission-line model represents more closely the lumped element.

Consider the lumped network shown in Fig. 2(a). It is composed of $M$ simple resistive networks with scattering matrices $S_{1}, S_{2}, \ldots, S_{M}$, connected either by a simple pair of wires or a pair of wires containing a series inductor or a shunt capacitor or both. In the transmission-line model these connections are replaced by transmission-line sections of propagation time $T$ as shown in Fig. 2(b). In this casc the model is called a link transmission-line model (3).

The numerical method operates by considering a pulse to be injected into the input terminals of the whole network. The pulse scatters on reaching the first sub-network being partly reflected and partly transmitted. This scattering occurs at every sub-network, pulses racing to and fro between sub-networks. The output impulse function is the stream of pulses at the output terminals. 
J. W. Bandler, P. B. Johns and M. R. M. Rizk

(a)

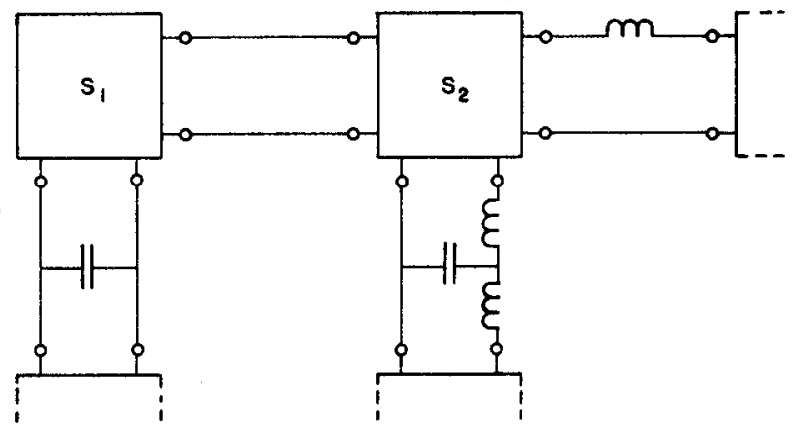

(b)

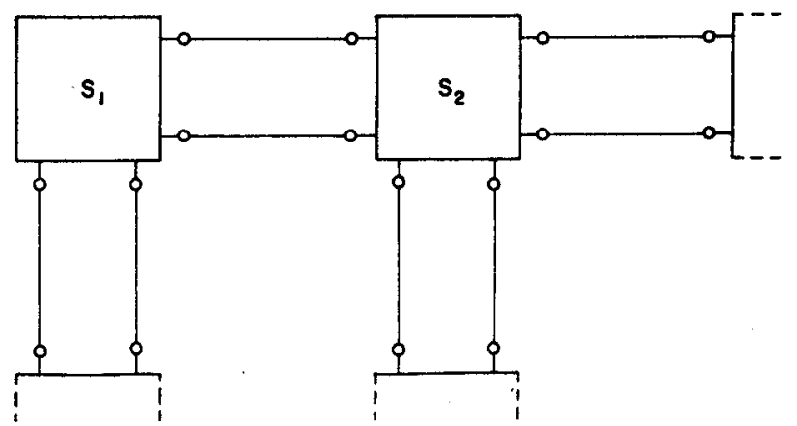

FIG. 2. Lumped network and link transmission-line model.

If the $m$ th network has $N$ ports with incident and reflected voltages given by (3)

$$
\mathbf{V}_{m}^{i}=\left[\begin{array}{c}
V_{m 1}^{i} \\
V_{m 2}^{i} \\
\cdot \\
\cdot \\
\cdot \\
V_{m N}^{i}
\end{array}\right], \quad \mathbf{V}_{m}^{r}=\left[\begin{array}{c}
V_{m 1}^{r} \\
V_{m 2}^{r} \\
\cdot \\
\cdot \\
V_{m N}^{r}
\end{array}\right]
$$

then the scattering equation is

$$
{ }_{k} \mathbf{V}_{m}^{r}=\mathbf{S}_{m k} \mathbf{V}_{m}^{i},
$$

where the subscript $k$ denotes the $k$ th time step. If all the incident and reflected pulses are assembled into the partitioned vectors

$$
\mathbf{V}^{i}=\left[\begin{array}{c}
\mathbf{V}_{1}^{i} \\
\mathbf{V}_{2}^{i} \\
\cdot \\
\cdot \\
\cdot \\
\mathbf{V}_{M}^{i}
\end{array}\right], \quad \mathbf{V}^{\prime}=\left[\begin{array}{c}
\mathbf{V}_{1}^{r} \\
\mathbf{V}_{2}^{r} \\
\cdot \\
\cdot \\
\cdot \\
\mathbf{V}_{M}^{r}
\end{array}\right]
$$


then the scattering equation for the entire network is

$$
{ }_{k} \mathbf{V}^{r}=\mathbf{S}_{k} \mathbf{V}^{i}
$$

where $\mathbf{S}$ in this case is a block diagonal partitioned matrix with $\mathbf{S}_{1}, \mathbf{S}_{2}, \ldots, \mathbf{S}_{M}$ on the diagonal.

The reflected pulses are the incident pulses at the next time step, and they are related by

$$
{ }_{k+1} \mathbf{V}^{i}=\mathbf{C}_{k} \mathbf{V}^{r}
$$

where $\mathbf{C}$ is the connection matrix indicating the transmission of reflected pulses from one sub-network to become incident pulses on a neighbouring subnetwork. The iteration equation is

$$
{ }_{k+1} \mathbf{V}^{i}=\operatorname{CS}_{k} \mathbf{V}^{i}
$$

The method will be unconditionally stable for a passive RLC lumped network and, therefore, it will be useful for stiff networks (3).

\section{Stub Modeling}

A lumped network consisting of resistive, inductive and capacitive elements may also be modeled by stub transmission lines. In this case, the time taken by a pulse to travel to the end of the stub and back again is T. Following the same procedure used in the link transmission-line models, an inductor is modeled by a short-circuit stub with characteristic impedance

$$
Z_{0}=\frac{2 L}{T}
$$

and the modeling error is a capacitor given by

$$
C=\frac{T^{2}}{4 L}
$$

A capacitor is modeled by an open-circuit stub with an impedance

$$
Z_{0}=\frac{T}{2 C}
$$

and the modeling error is an inductor given by

$$
L=\frac{T^{2}}{4 C} \text {. }
$$

The elements with these models are shown in Fig. 3.

Consider the lumped network in Fig. 4(a), which is represented by a resistive network with $N$ pairs of terminals to which all of the inductors and capacitors are connected as shown. A transmission-line model of the circuit is shown in Fig. 4(b) in which all of the inductors are replaced by open-circuit stubs. The 
J. W. Bandler, P. B. Johns and M. R. M. Rizk

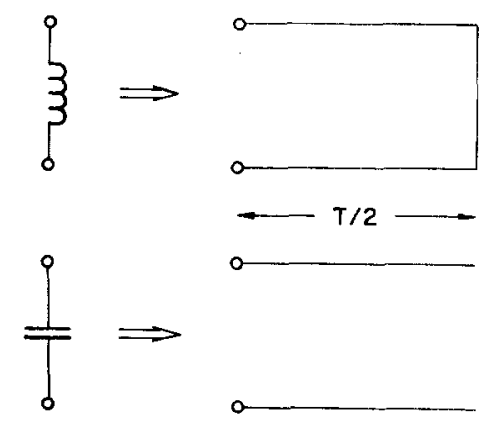

Fig. 3. Stub models of an inductor and a capacitor.

reflected pulses

$$
\mathbf{V}^{r}=\left[\begin{array}{c}
V_{1}^{r} \\
V_{2}^{r} \\
\cdot \\
\cdot \\
V_{N}^{r}
\end{array}\right]
$$

will be scattered instantaneously into the $N$ stubs. These pulses will travel to the ends of the stubs and be reflected or reflected and inverted for capacitive or inductive stubs, respectively. The pulses then return to the resistive network and become incident pulses

$$
\mathbf{V}^{i}=\left[\begin{array}{c}
V_{1}^{i} \\
V_{2}^{i} \\
\cdot \\
\cdot \\
\cdot \\
V_{N}^{i}
\end{array}\right]
$$

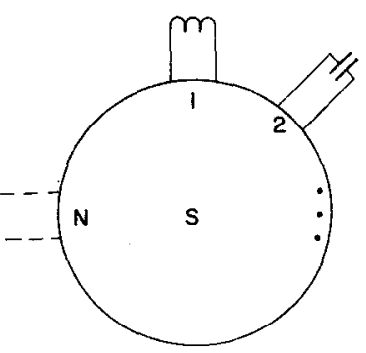

(a)

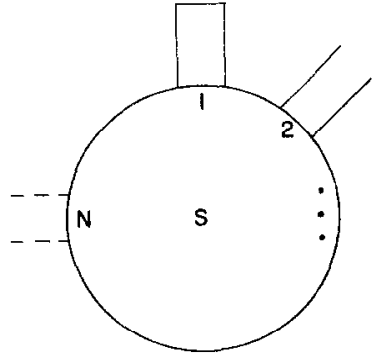

(b)

Fig. 4. Lumped network and transmission-line stub model. 
If the scattering matrix of the resistive network is the $N \times N$ matrix $\mathbf{S}$ then, at the $k$ th iteration,

$$
{ }_{k} \mathbf{V}^{r}=\mathbf{S}_{k} \mathbf{V}^{i} \text {. }
$$

Reflection of the pulses at the end of the stubs gives the incident pulses at time $k+1$, obtained using the same formula as (12), where $\mathbf{C}$, in this case, is an $N \times N$ diagonal matrix with an entry of 1 for a capacitive stub and -1 for an inductive stub. The iteration routine is therefore exactly as (13).

To enable the incident pulses $\mathbf{V}^{i}$ to converge simultaneously it is sufficient that the propagation time $T$ be the same for all the stubs. This propagation time is therefore the same as the iteration time. This method is also unconditionally stable for a lumped network of positive resistors, inductors and capacitors.

\section{Discussion}

It should be noted that the stub modeling leads to an implicit routine. The reason is that the scattering matrix $\mathbf{S}$ involves the entire resistive network. Thus, to calculate $\mathbf{S}$ it is necessary to invert a set of simultaneous equations describing the network. If the network is nonlinear, then this inversion is required before every iteration. In link transmission-line modeling, however, the iteration routine is explicit, the complexity of equations being independent of the number of sub-networks or nodes. The scattering matrices of the networks are small enough to be calculated by simple formulas, for example, the scattering matrix of the sub-network in Fig. 5(a) is given by

$$
\begin{aligned}
& \frac{1}{R+Z_{1}+Z_{2}} \\
& \quad \times\left[\begin{array}{cc}
\frac{R\left(R+Z_{1}+Z_{2}\right)+2 Z_{1}\left(Z_{2}-Z_{1}\right)}{R+2 Z_{1}} & 2 Z_{1} \\
2 Z_{2} & \frac{R\left(R+Z_{1}+Z_{2}\right)+2 Z_{2}\left(Z_{1}-Z_{2}\right)}{R+2 Z_{2}}
\end{array}\right]
\end{aligned}
$$

and the scattering matrix of the sub-network in Fig. 5(b) is

$$
\begin{aligned}
& \frac{1}{Z_{1} Z_{2}+Z_{1} Z_{3}+Z_{2} Z_{3}} \\
& \quad \times\left[\begin{array}{ccc}
Z_{2} Z_{3}-Z_{1} Z_{2}-Z_{3} Z_{1} & 2 Z_{1} Z_{3} & 2 Z_{1} Z_{2} \\
2 Z_{2} Z_{3} & Z_{1} Z_{3}-Z_{1} Z_{2}-Z_{2} Z_{3} & 2 Z_{1} Z_{2} \\
2 Z_{2} Z_{3} & 2 Z_{1} Z_{3} & Z_{1} Z_{2}-Z_{1} Z_{3}-Z_{3} Z_{2}
\end{array}\right]
\end{aligned}
$$

In general, a network may be modeled by either one or both types of model. The LC lowpass filter in Fig. 6(a) can have the link model of Fig. 6(b) or the stub model of Fig. 6(c) or the mixed model of Fig. 6(d).

\section{Example}

The following example illustrates the TLM routine for link modeling. Consider the circuit of Fig. 6(a) (4) and its link transmission-line model in Fig. 6(b). 
J. W. Bandler, P. B. Johns and M. R. M. Rizk

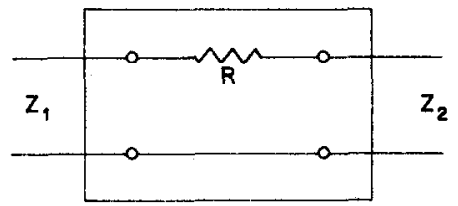

(a)

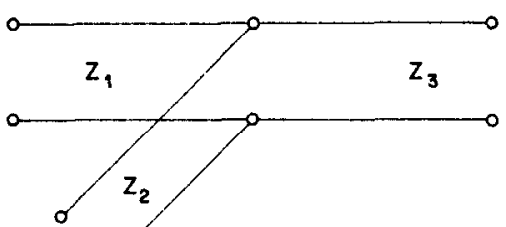

(b)

Fig. 5. Example of two simple sub-networks.

(a)

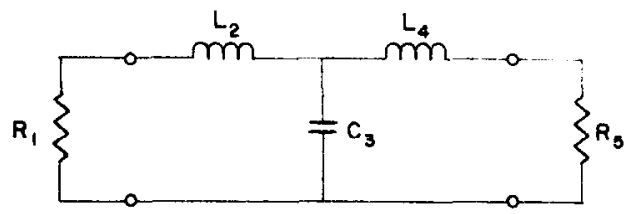

(b)
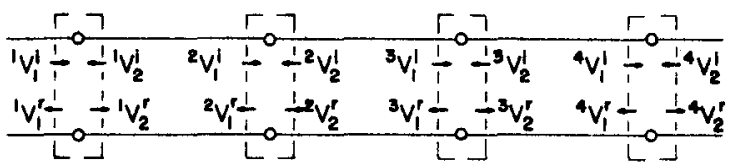

(c)

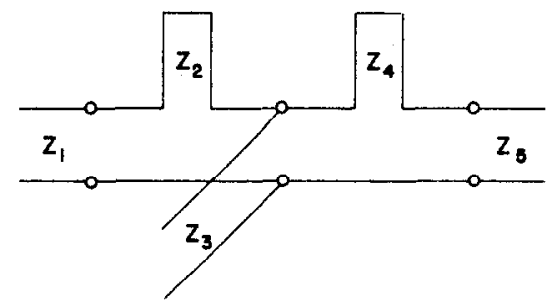

(d)

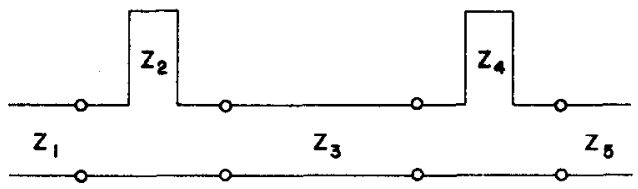

FIG. 6. LC lowpass filter and different types of models. (a) the filter, (b) link model, (c) stub model, (d) mixed model. 
TABLE I

Incident and Reflected Pulses of the Circuit in Fig. 6(b)

\begin{tabular}{cccccccc}
\hline $\begin{array}{c}t \\
(\mathrm{sec})\end{array}$ & ${ }^{1} V_{1}^{\mathrm{i}}$ & ${ }^{1} V_{2}^{\mathrm{i}}$ & ${ }^{1} V_{2}^{r}$ & ${ }^{2} V_{1}^{i}$ & ${ }^{2} V_{1}^{r}$ & ${ }^{2} V_{2}^{i}$ & ${ }^{2} V_{2}^{r}$ \\
\hline 0.0 & 0.5 & - & 0.90909 & - & - & - & - \\
0.1 & - & - & - & 0.90909 & -0.90004 & - & 0.00904 \\
0.2 & - & -0.90004 & 0.73639 & - & - & - & - \\
0.3 & - & - & - & 0.73639 & -0.71125 & 0.00895 & 0.01619 \\
0.4 & - & -0.71125 & 0.58193 & - & - & - & - \\
0.5 & - & - & - & 0.58193 & -0.54452 & 0.01589 & 0.02151 \\
\hline & & & & & & & \\
\hline$t$ & & & & & & & \\
$(\mathrm{sec})$ & ${ }^{3} V_{1}^{i}$ & ${ }^{3} V_{1}^{r}$ & ${ }^{3} V_{2}^{i}$ & ${ }^{3} V_{2}^{r}$ & ${ }^{4} V_{1}^{i}$ & ${ }^{4} V_{1}^{r}$ & ${ }^{4} V_{2}^{r}$ \\
\hline 0.0 & - & - & - & - & - & - & - \\
0.1 & - & - & - & - & - & - & - \\
0.2 & 0.00904 & 0.00895 & - & 0.01800 & - & - & - \\
0.3 & - & - & - & - & 0.01800 & -0.01472 & 0.00327 \\
0.4 & 0.01619 & 0.01589 & -0.01472 & 0.04680 & - & - & - \\
0.5 & - & - & - & - & 0.04680 & -0.03829 & 0.00851 \\
\hline
\end{tabular}

Let the time step $T$ be 0.1 second and the component values $L_{2}=L_{4}=1$, $C_{3}=2$ and $R_{1}=R_{5}=1$. The characteristic impedances $Z_{2}, Z_{3}$ and $Z_{4}$ are 10 , 0.05 and 10 , respectively. An incident pulse of value 0.5 is launched into the transmission line representing the source resistance and hits the first junction at time $t=0$. The pulse scatters producing reflected and transmitted pulses. The transmitted pulses travel towards the output, being scattered at the other junctions. The pulses propagate forwards and backwards between the junctions. Table I gives the incident and reflected pulses at the junctions of Fig. 6(b) at different times.

\section{Compensation of Errors}

Errors in the TLM method arise only from how well the transmission-line model represents the actual circuit. Errors do not arise from the numerical solution of the model. In certain cases the unwanted distributed elements are reduced when the step size $T$ is reduced. A distributed capacitor in modeling an inductor is an example of such a case. We have to note that this capacitor is known before any calculation is started, since $T$ has to be chosen. If the distributed error capacitor is taken to be lumped and half placed at each end of the transmission-line, each of these lumped capacitors has a value of $C_{d} l / 2$. The inductor and the two capacitors representing modeling errors are shown in Fig. 7. To compensate for modeling error (to some degree) we can subtract the error capacitor from the original neighbouring network components. As $T$ increases the amount to be subtracted increases and it becomes obvious that there may be a limit to such compensation. 
J. W. Bandler, P. B. Johns and M. R. M. Rizk

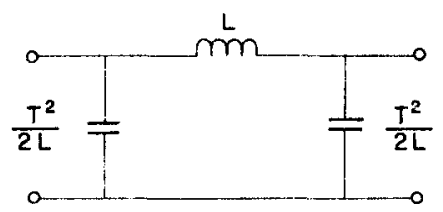

Fig. 7. An inductor with two capacitors representing modeling error.

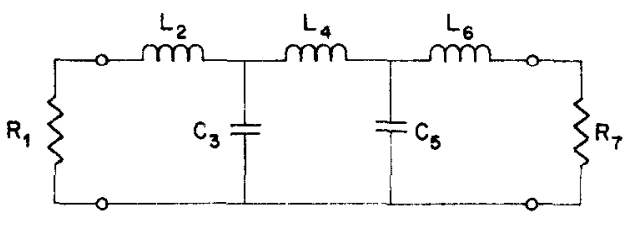

Fig. 8. Chebyshev filter with 7 elements.

The impulse response of the Chebyshev filter shown in Fig. 8 (5) was found by Kutta-Simpson, Euler, TLM and TLM with compensation. The results are shown in Table II. The advantage of compensation is clear from the table comparing the percentage error between the Kutta-Simpson integration method and the other methods. The actual components and the new components after compensation are given in Table III.

TABLE II

Comparison Between Different Methods of Integration and TLM Modeling With and Without Compensation

\begin{tabular}{crrcc}
\hline & & \multicolumn{3}{c}{ Percentage error for $T=0.1$} \\
\cline { 3 - 5 } $\begin{array}{c}t \\
\text { (sec) }\end{array}$ & Kutta-Simpson & Euler & Link modeling & $\begin{array}{c}\text { Link modeling } \\
\text { with compensation }\end{array}$ \\
\hline 1.1 & 0.003981 & -38.5 & -8.3 & -6.9 \\
2.1 & 0.035665 & -12.8 & -2.3 & -1.4 \\
3.1 & 0.101499 & 0.0 & -0.9 & 0.1 \\
4.1 & 0.160644 & 8.2 & -0.3 & 0.3 \\
5.1 & 0.161516 & 13.1 & 0.3 & 0.2 \\
6.1 & 0.094384 & 11.1 & 1.3 & -0.3 \\
7.1 & 0.002772 & 520.3 & 48.9 & -21.8 \\
8.1 & -0.054462 & 57.9 & -1.2 & 0.5 \\
9.1 & -0.051167 & 45.6 & 0.7 & -0.7 \\
\hline
\end{tabular}

\section{Sensitivity Evaluation}

One of the features of the TLM method is that simple calculation of exact sensitivities w.r.t. design variables is possible. Sensitivities are calculated iteratively in the same iteration process for calculating the impulse response. 
Transmission-Line Modeling and Sensitivity Evaluation

TABLE III

Component Values of the Filter Shown in Fig. 8 Before and After Compensation

\begin{tabular}{lcccccccc}
\hline \multicolumn{8}{c}{ Component values in Ohms, Henries and Farads } \\
\hline \multicolumn{1}{c}{ State } & $R_{1}$ & $L_{2}$ & $C_{3}$ & $L_{4}$ & $C_{5}$ & $L_{6}$ & $R_{7}$ \\
\hline No compensation & 1.0 & 1.7058 & 1.2296 & 2.5408 & 1.2296 & 1.7058 & 1.0 \\
With compensation & 1.0 & 1.7017 & 1.2247 & 2.5327 & 1.2247 & 1.7017 & 1.0 \\
\hline
\end{tabular}

\section{First-Order Sensitivities}

Equation (11) or (20) describe the relationship between appropriate incident and reflected voltages for the whole network and the derivatives w.r.t. the $\kappa$ parameters of the whole network can be written as

$$
\left[\begin{array}{c}
{ }_{k} \mathbf{V}^{\prime} \\
\frac{\partial_{k} \mathbf{V}^{r}}{\partial \phi_{1}} \\
\cdot \\
\cdot \\
\frac{\partial_{k} \mathbf{V}^{r}}{\partial \phi_{k}}
\end{array}\right]=\left[\begin{array}{cccc}
\mathbf{S} & \mathbf{0} & \ldots & 0 \\
\frac{\partial \mathbf{S}}{\partial \phi_{1}} & \mathbf{S} & & \mathbf{0} \\
\cdot & & \cdot & \\
\cdot & \mathbf{0} & \cdot & \mathbf{0} \\
\cdot & & \cdot & \\
\frac{\partial \mathbf{S}}{\partial \phi_{\kappa}} & \mathbf{0} & & \mathbf{S}
\end{array}\right]\left[\begin{array}{c}
{ }_{k} \mathbf{V}^{i} \\
\frac{\partial_{k} \mathbf{V}^{i}}{\partial \phi_{1}} \\
\cdot \\
\cdot \\
\cdot \\
\frac{\partial_{k} \mathbf{V}^{i}}{\partial \phi_{k}}
\end{array}\right] .
$$

The right-hand side vector is obtained from an equation of the form of (12) after differentiating it w.r.t. the $j$ th parameter, viz.,

$$
\frac{\partial_{k} \mathbf{V}^{i}}{\partial \phi_{j}}=\mathbf{C}{\frac{\partial}{\partial \phi_{j}}}^{k-1} \mathbf{V}^{r}
$$

where $\mathbf{C}$ is constant.

It is clear that the matrix in (23) is very sparse since, for example, $\partial \mathbf{S}_{m} / \partial \phi_{j}$ vanishes if $\mathbf{S}_{m}$ does not contain the $j$ th parameter. Although this matrix is sparse, the two vectors on the left and right hand sides are full and all the information has to be transferred in each iteration. So in calculating the sensitivities, we have to find the sensitivity of all the incident pulses w.r.t. all the parameters. The sensitivity of the impulse response will be the sensitivity of the stream of pulses at the output port w.r.t. the parameters.

Consider a sub-network which simply connects two transmission lines having $Z_{1}$ and $Z_{2}$ as their characteristic impedances. The scattering matrix $\mathbf{S}_{m}$ is given by

$$
\mathbf{S}_{m}=\frac{1}{Z_{1}+Z_{2}}\left[\begin{array}{cc}
Z_{2}-Z_{1} & 2 Z_{1} \\
2 Z_{2} & Z_{1}-Z_{2}
\end{array}\right]
$$


Let

$$
Z_{1}=\phi_{j}, \quad Z_{2}=\phi_{j+1}
$$

Then

$$
\begin{aligned}
\frac{\partial \mathbf{S}_{m}}{\partial \phi_{j}} & =\frac{2 Z_{2}}{\left(Z_{1}+Z_{2}\right)^{2}}\left[\begin{array}{rr}
-1 & 1 \\
-1 & 1
\end{array}\right] \\
\frac{\partial \mathbf{S}_{m}}{\partial \phi_{j+1}} & =\frac{2 Z_{1}}{\left(Z_{1}+Z_{2}\right)^{2}}\left[\begin{array}{rr}
1 & -1 \\
1 & -1
\end{array}\right] .
\end{aligned}
$$

The expressions (25)-(27) can be fitted into the scheme of (23) for this sub-network.

\section{Second-Order Sensitivities}

Differentiating (11) or (20) w.r.t. the $j$ th parameter, we get

$$
\frac{\partial_{k} \mathbf{V}^{r}}{\partial \phi_{j}}=\frac{\partial \mathbf{S}}{\partial \phi_{j}}{ }_{k} \mathbf{V}^{i}+\mathbf{S} \frac{\partial_{k} \mathbf{V}^{i}}{\partial \phi_{j}}
$$

where $\partial_{k} \mathbf{V}^{i} / \partial \phi_{j}$ is found from (24).

If we differentiate (24) and (28) w.r.t. $\phi_{l}$ we get, respectively,

$$
\begin{gathered}
\frac{\partial^{2}{ }_{k} \mathbf{V}^{i}}{\partial \phi_{l} \partial \phi_{j}}=\mathbf{C} \frac{\partial^{2}{ }_{k-1} \mathbf{V}^{i}}{\partial \phi_{l} \partial \phi_{i}} \\
\frac{\partial^{2}{ }_{k} \mathbf{V}^{r}}{\partial \phi_{l} \partial \phi_{i}}=\frac{\partial^{2} \mathbf{S}}{\partial \phi_{l} \partial \phi_{j}}{ }_{k} \mathbf{V}^{i}+\frac{\partial \mathbf{S}}{\partial \phi_{j}} \frac{\partial_{k} \mathbf{V}^{i}}{\partial \phi_{l}}+\frac{\partial \mathbf{S}}{\partial \phi_{l}} \frac{\partial_{k} \mathbf{V}^{i}}{\partial \phi_{j}}+\mathbf{S} \frac{\partial^{2}{ }_{k} \mathbf{V}^{i}}{\partial \phi_{l} \partial \phi_{j}}
\end{gathered}
$$

Equation (30) holds for sub-networks when subscript $m$ is applied to both sides but some of the derivatives of $\mathbf{S}_{m}$ are zero.

\section{Examples}

The symmetrical $L C$ lowpass filter shown in Fig. 6(a) has been optimized in the time domain. Figure 9 shows a specified impulse response for $L_{2}=L_{4}=1.0$, $C_{3}=2.0$. Taking 100 sample points, using TLM analysis, least 4 th approximation yielded the solution in $21 \mathrm{~s}$ ( 24 function evaluations) and $17 \mathrm{~s}$ (19 function evaluations) from starting points $a$ and $b$, respectively, with a maximum error of about $3 \times 10^{-7}$. The specifications of Fig. 10 were met with a minimax error of 0.0021992 after $37 \mathrm{~s}$ (46 function evaluations) using 33 sample points for optimization. The starting point was $L_{2}=L_{4}=C_{3}=1.0$ and the optimum point reached was $L_{2}=L_{4}=0.76645547$ and $C_{3}=2.3739403$. The minimax solution was reached using third-order extrapolation, after a sequence of least $p$ th optimizations where the values of $p$ were $4,16,64,256$ and 1024. FLOPT2, a program described in (6), was used in these examples. The computer was a CDC 6400. 


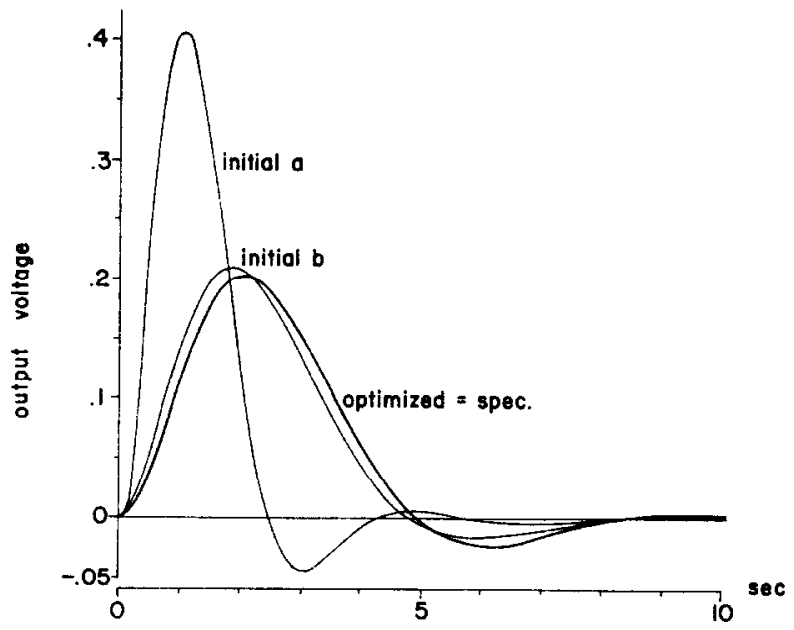

FIg. 9. Optimization using TLM analysis. Starting point a: $L_{2}=L_{4}=0.5, C_{3}=1.0$. Starting point b: $L_{2}=L_{4}=0.8, C_{3}=2.2$.

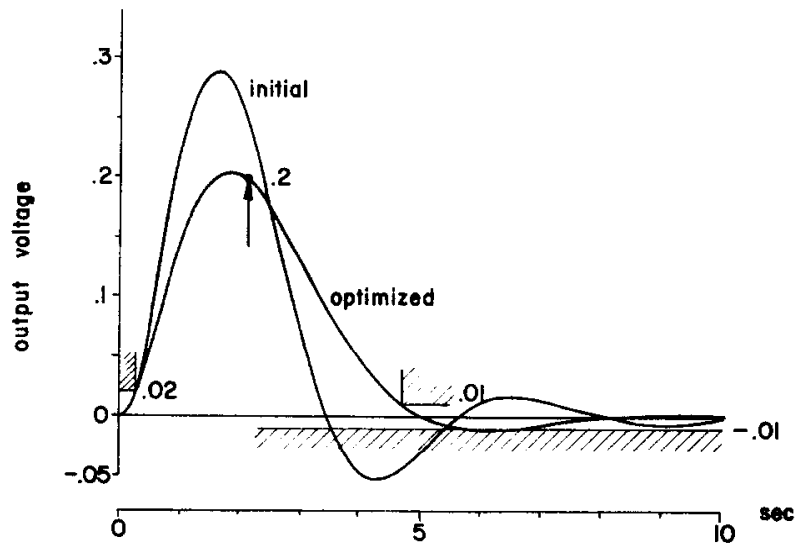

FIG. 10. Optimization using TLM analysis. Starting point $L_{2}=L_{4}=C_{3}=1.0$. Solution: $L_{2}=L_{4}=0.76646, C_{3}=2.3739$.

V. Sensitivities with Respect to Time and $T$

Differentiating (11) or (20) w.r.t. $T$ we get

$$
\frac{\partial_{k} \mathbf{V}^{r}}{\partial T}=\frac{\partial \mathbf{S}}{\partial T}{ }_{k} \mathbf{V}^{i}+\mathbf{S} \frac{\partial_{k} \mathbf{V}^{i}}{\partial T}
$$


Usually the scattering matrix $\mathbf{S}$ includes the parameters $\boldsymbol{\phi}$ which are functions of $T$ as obtained from the modeling.

The term $\partial \mathrm{S} / \partial T$ can be obtained from

$$
\frac{\partial \mathbf{S}}{\partial T}=\sum_{i} \frac{\partial \mathbf{S}}{\partial \phi_{j}} \frac{\partial \phi_{j}}{\partial T} .
$$

Suppose $\phi_{j}$ is the characteristic impedance of a line modeling an inductor. If $\phi_{j}=L / T$ then $\partial \phi_{j} / \partial T=-\phi_{j} / T$. For the capacitive case, $\partial \phi_{j} / \partial T=\phi_{j} / T$. The second term on the right-hand side of (31) is obtained from (12), where

$$
\frac{\partial_{k} \mathbf{V}^{i}}{\partial T}=\mathbf{C} \frac{\partial}{\partial T}_{k-1} \mathbf{V}^{r}
$$

Note that the differentiation is at discrete time steps and the information is transferred iteratively with the original iteration scheme of the TLM method. Thus the above derivatives can only be obtained at points corresponding to fixed numbers of iterations $k$, i.e., at $t=k T$, where $t$ is time. Let $f(t, T)$, be an interpolation to the approximation of the impulse response obtained at discrete times $t_{1}, t_{2}, t_{3}, \ldots$ by the TLM method, where

$$
t_{j}-t_{j-1}=n T
$$

where $n$ is an integer.

The parameter $T$ is chosen arbitrarily, although it is known that the smaller the $T$ the more accurate is the modeling.

Suppose that the analysis is done twice with two different time steps $T_{1}$ and $T_{2}$, respectively. In the first analysis we will get $f\left(t, T_{1}\right)$ at points, in general, time $n T_{1}$ apart, and in the second analysis $f\left(t, T_{2}\right)$ at points $n T_{2}$ apart. Figure 11 illustrates the situation.

A first-order change in $f(t, T)$ is given by

$$
\delta f=\frac{\partial f}{\partial t} \Delta t+\frac{\partial f}{\partial T} \Delta T
$$

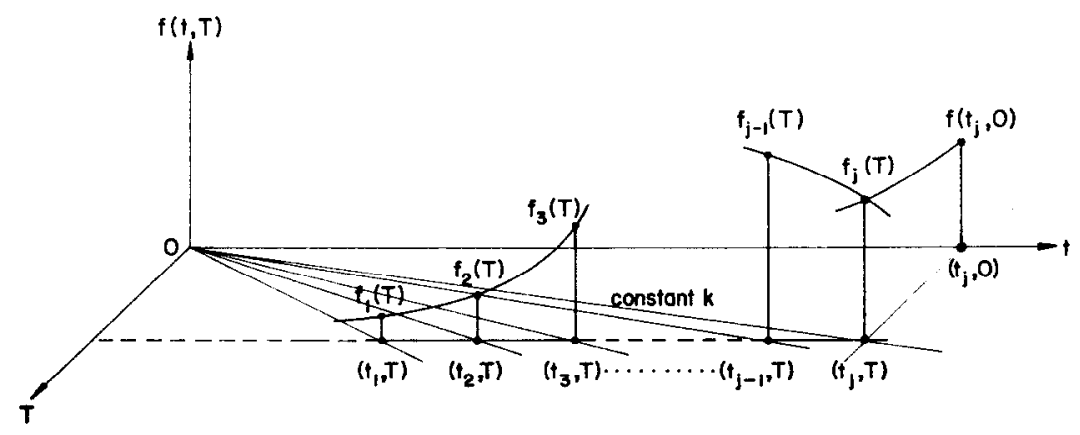

FiG. 11. Representation of response with respect to $t$ and $T$. 
Transmission-Line Modeling and Sensitivity Evaluation

TABLE IV

$\left.(1 / k)(\partial f / \partial T)\right|_{k}$ Obtained from the TLM Routine Versus af/at by Central Differences Where $T=0.1$

\begin{tabular}{cccc}
\hline $\begin{array}{c}t \\
(\mathrm{sec})\end{array}$ & $\left.\frac{1}{k} \frac{\partial f}{\partial T}\right|_{k}$ & $\begin{array}{c}\frac{\partial f}{\partial t} \\
\text { (central differences) }\end{array}$ & $\begin{array}{c}\text { Difference } \\
(\%)\end{array}$ \\
\hline 0.5 & 0.13999 & 0.14638 & 4.56 \\
1.1 & 0.13649 & 0.13771 & 0.89 \\
1.7 & 0.05300 & 0.05315 & 0.28 \\
2.3 & -0.03086 & -0.03101 & 0.48 \\
2.9 & -0.08180 & -0.08206 & 1.54 \\
3.5 & -0.09538 & -0.09569 & 0.32 \\
4.1 & -0.08170 & -0.08202 & 0.39 \\
4.7 & -0.05493 & -0.05521 & 0.51 \\
5.3 & -0.02705 & -0.02727 & 0.81 \\
5.9 & -0.00535 & -0.00549 & 2.62 \\
\hline
\end{tabular}

where $\Delta t$ and $\Delta T$ are changes in $t$ and $T$, respectively. Thus,

$$
\frac{\delta f}{\Delta T}=\frac{\partial f}{\partial t} \frac{\Delta t}{\Delta T}+\frac{\partial f}{\partial T}
$$

From the relation $t=k T$ we have

$$
\Delta t=k \Delta T
$$

therefore, for a particular $k$

$$
\left.\frac{\delta f}{\Delta T}\right|_{\Delta r \rightarrow 0}=\left.\frac{\partial f}{\partial T}\right|_{k}=k \frac{\partial f}{\partial t}+\frac{\partial f}{\partial T} .
$$

The term $\partial f /\left.\partial T\right|_{k}$ is obtained from (31).

Table IV shows, for the circuit of Fig. $6(\mathrm{a})$, where $f_{j}(T)={ }_{k}^{4} \mathbf{V}_{2}^{r} / 2 T,(1 / k)$ $\left.(\partial f / \partial T)\right|_{k}$ versus $\partial f / \partial t$ calculated by the central difference formula given in the appendix. There is a difference between the numbers in the two columns which we can attribute to $(1 / k)(\partial f / \partial T)$ and the inexactness of calculating $\partial f / \partial t$. However, it is clear that this difference is very small.

Table $V$ compares the results obtained for $\partial f /\left.\partial T\right|_{k}$ obtained from the TLM routine, and the ones obtained by perturbing $T$ to 0.101 and 0.099 from its initial value 0.1 (i.e., repeat the analysis with these new values of $T$ ), and using central differences.

Two analyses were performed with two different time steps, namely, 0.1 and $0.5 / 7$, and $\partial f / \partial T$ at constant time was estimated by perturbation as $\Delta f / \Delta T$. This $\Delta f / \Delta T$ was used to extrapolate to the exact response. The extrapolation formula

$$
f_{\text {extrapolated }}=f_{\mathrm{TLM}}-\frac{T}{2} \frac{\Delta f}{\Delta T}
$$


TABLE V

A Comparison Between $\partial f / \partial T$ For Constant $k$ Obtained by TLM and Perturbation

\begin{tabular}{|c|c|c|c|c|c|}
\hline \multirow{2}{*}{$k$} & $f(t$, & & \multirow{2}{*}{$\begin{array}{c}\left.\frac{\partial f}{\partial T}\right|_{k} \\
\text { (central } \\
\text { differences) }\end{array}$} & \multirow{2}{*}{$\left.\frac{\partial f}{\partial T}\right|_{k}$} & \multirow{2}{*}{$\begin{array}{c}\text { Difference } \\
(\%)\end{array}$} \\
\hline & $T=0.101$ & $T=0.099$ & & & \\
\hline 11 & 0.13581327 & 0.13281062 & 1.50132 & 1.50137 & 0.006 \\
\hline 41 & 0.04989793 & 0.05659663 & -3.34935 & -3.34976 & 0.019 \\
\hline 71 & -0.01400295 & -0.01576903 & 0.88304 & 0.88397 & 0.105 \\
\hline 101 & 0.00345630 & 0.00371329 & -0.12850 & -0.12911 & 0.474 \\
\hline 131 & -0.00061299 & -0.00057152 & -0.02073 & -0.02050 & 1.109 \\
\hline
\end{tabular}

Table VI compares the exact response obtained by the inverse Laplace transform and the extrapolated response. Table VII, on the other hand, compares the exact response and the extrapolated one, where $\partial f / \partial T$ was calculated using (38) for which $\partial f / \partial t$ is calculated by the central difference formula given in the appendix.

$$
\text { TABLe VI }
$$

Using $\Delta f / \Delta T$ to Predict Response for $T=0$

\begin{tabular}{ccccccc}
\hline \multirow{2}{*}{$\begin{array}{c}\boldsymbol{c}(t, T) \\
\text { (sec) }\end{array}$} & $T=0.1$ & $T=0.5 / 7$ & $\frac{\Delta f}{\Delta T}$ & $f_{\text {exact }}$ & $f_{\text {extrapolated }}$ & $\begin{array}{c}\text { Difference } \\
(\%)\end{array}$ \\
\hline 0.5 & 0.04255324 & 0.04333199 & $-2.72562 \times 10^{-2}$ & 0.044141 & 0.043916 & 0.510 \\
1.5 & 0.17926209 & 0.17938925 & $-4.21435 \times 10^{-3}$ & 0.179524 & 0.179473 & 0.028 \\
2.5 & 0.19006031 & 0.18991132 & $4.51465 \times 10^{-3}$ & 0.189777 & 0.189815 & 0.020 \\
3.5 & 0.10754101 & 0.10727105 & $9.44860 \times 10^{-3}$ & 0.106988 & 0.107069 & 0.076 \\
4.5 & 0.02373052 & 0.02339983 & $1.15742 \times 10^{-2}$ & 0.023053 & 0.023152 & 0.429 \\
\hline
\end{tabular}

TABLE VII

Using $\partial f / \partial T$ to Predict Response for $T=0$

\begin{tabular}{cccc}
\hline $\begin{array}{c}t \\
(\mathrm{sec})\end{array}$ & \multicolumn{1}{c}{$f_{\text {exact }}$} & $f_{\text {extrapolated }}$ & $\begin{array}{c}\text { Difference } \\
(\%)\end{array}$ \\
\hline 0.5 & 0.044141 & 0.044152 & 0.014 \\
1.1 & 0.134981 & 0.134992 & 0.008 \\
1.7 & 0.193099 & 0.193111 & 0.006 \\
2.3 & 0.198260 & 0.198272 & 0.006 \\
2.9 & 0.162173 & 0.162183 & 0.006 \\
3.5 & 0.106988 & 0.106994 & 0.006 \\
4.1 & 0.052558 & 0.052561 & 0.006 \\
4.7 & 0.011055 & 0.011054 & 0.009 \\
5.3 & -0.013432 & -0.013435 & 0.022 \\
5.9 & -0.022686 & -0.022690 & 0.017 \\
\hline
\end{tabular}




\section{Conclusions}

The TLM method is a new approach to the analysis of lumped networks. The distinct advantage of the TLM method is that the numerical procedure used solves the transmission-line model exactly. Errors arise only from how well the transmission-line model represents the actual circuit. To a certain limit the compensation of these errors by additional elements yielded more accurate results.

Another advantage is that if the transmission-line network is physically stable, which is true in the case of passive linear networks, then the TLM solution will be stable. This means that stiff networks which give rise to instability in most methods do not cause instability in the TLM method. Different transmission-line models can be obtained for the same network, some of the models can be viewed as implicit methods and some as explicit.

The simplicity of calculating the sensitivity of the impulse response with respect to design parameters makes the TLM method suitable for automated network design. Sensitivities with respect to time and time step can be easily obtained and it has been demonstrated how this information is used to improve accuracy.

Possible developments in the method lie in improving the accuracy by using more complicated transmission-line elements and models and the investigation of limitations on modeling general sets of coupled ordinary differential equations.

\section{References}

(1) S. Akhtarzad and P. B. Johns, "The solution of Maxwell's equations in three space dimensions and time by the TLM method of numerical analysis", Proc. IEE, Vol. 122, pp. $1349-1352$, Dec. 1975.

(2) P. B. Johns, "Solution of the lossy wave equation by the TLM method", Proc. AICA Int. Symp. Computer Methods for Partial Differential Equations Bethlehem, Pa., pp. 247-250, June 1975.

(3) P. B. Johns, "Numerical modelling by the TLM method", Proc. Int. Symp. Large Engineering Systems Winnipeg, Canada, Aug. 1976.

(4) J. W. Bandler, H. L. Abdel-Malek, P. B. Johns and M. R. M. Rizk, "Optimal design via modeling and approximation", Proc. IEEE Int. Symp. Circuits and Systems Munich, pp. 767-770, Apr. 1976.

(5) G. L. Matthaei, L. Young and E. M. T. Jones, "Microwave Filters Impedancematching Networks, and Coupling Structures", McGraw-Hill, New York, 1964.

(6) J. W. Bandler and W. Y. Chu, "FLOPT2-a program for least pth optimization with extrapolation to minimax solutions", IEEE Trans. Microwave Theory Tech., Vol. MTT-24, pp. 543-544, Aug. 1976.

(7) L. G. Kelly, "Handbook of Numerical Methods and Applications", AddisonWesley, New York, 1967.

\section{Appendix}

The formula used to obtain $\partial f / \partial t$ from the response is (7)

$$
h f_{j}^{\prime}=\left(\mu \delta-\frac{1}{6} \mu \delta^{3}\right) f_{j}
$$


J. W. Bandler, P. B. Johns and M. R. M. Rizk

where

$$
\mu f(t)=\frac{1}{2}\left[f\left(t+\frac{h}{2}\right)+f\left(t-\frac{h}{2}\right)\right]
$$

and

$$
\delta f(t)=f\left(t+\frac{h}{2}\right)-f\left(t-\frac{h}{2}\right) .
$$

Equation (A1) can be rewritten as

$$
f_{j}^{\prime}=\frac{2}{3 h}[f(t+h)-f(t-h)]-\frac{1}{12 h}[f(t+2 h)-f(t-2 h)]
$$

In the tables given $h$ was equal to $2 T$. 